\title{
Implementation of the milestones communication approach for patients with limited prognosis: evaluation of intervention fidelity
}

Jasmin Bossert ${ }^{1 *}$, Michel Wensing $^{1}$, Michael Thomas ${ }^{2}$, Matthias Villalobos ${ }^{2}$, Corinna Jung $^{2}$, Anja Siegle ${ }^{2}$, Laura Hagelskamp², Nicole Deis ${ }^{3}$, Jana Jünger ${ }^{3}$ and Katja Krug ${ }^{1}$

\begin{abstract}
Background: Despite improvements in diagnostics and therapy, the majority of lung tumours are diagnosed at advanced stage IV with a poor prognosis. Due to the nature of an incurable disease, patients need to engage in shared decision making on advance care planning. To implement this in clinical practice, effective communication between patients, caregivers and healthcare professionals is essential. The Heidelberg Milestones Communication Approach (MCA) is delivered by a specifically trained interprofessional tandem and consists of four milestone conversations (MCs) at pivotal times in the disease trajectory. MC 1 (Diagnosis): i.e. prognosis; MC 2 (Stable disease): i.e. prognostic awareness; MC 3 (Progression): i.e. reassessment; MC 4 (Best supportive care): i.e. end of treatment. In between MCs, follow-up calls are carried out to sustain communication. This study aimed to assess to what extent the MCA was implemented as planned and consolidated in specialized oncology practice.
\end{abstract}

Methods: A prospective observational process evaluation study was conducted, which focused on the implementation fidelity of the MCA. All MCs during two assessment periods were included. We analysed all written records of the conversations, which are part of the routine documentation during MCs and follow-up calls. Adherence to key aspects of the manual was documented on structured checklists at the beginning of the implementation of the MCA and after 6 months. The analysis was descriptive. Differences between the two assessment periods are analysed with chi-square tests.

Results: A total of 133 MCs and 54 follow-up-calls (t1) and of 172 MCs and 92 follow-up calls (t2) were analysed. MC 2 were the most frequently completed conversations $(n=51$ and $n=47)$. Advance care planning was discussed in 26 and 13\% of MC 2 in the respective assessment periods; in 31 and 47\% of MC 2, prognostic awareness was recorded. The most frequently documented topic in the follow-up calls was the physical condition in patients (82 and $83 \%)$.

Conclusion: The implementation of a trajectory-specific communication concept was largely successful. Additional studies are needed to understand how fidelity could be further improved.

Trial registration: DRKS00013469 / Date of registration: 22.12.2017.

Keywords: Communication, Lung cancer, Advances care planning, Prognostic awareness, Patient preferences

\footnotetext{
* Correspondence: jasmin.bossert@med.uni-heidelberg.de

'Department of General Practice and Health Service Research, University

Hospital Heidelberg, Im Neuenheimer Feld 130.3, 69120 Heidelberg,

Germany

Full list of author information is available at the end of the article
}

(c) The Author(s). 2020 Open Access This article is distributed under the terms of the Creative Commons Attribution 4.0 International License (http://creativecommons.org/licenses/by/4.0/), which permits unrestricted use, distribution, and

reproduction in any medium, provided you give appropriate credit to the original author(s) and the source, provide a link to the Creative Commons license, and indicate if changes were made. The Creative Commons Public Domain Dedication waiver (http://creativecommons.org/publicdomain/zero/1.0/) applies to the data made available in this article, unless otherwise stated. 


\section{Background}

Lung cancer remains the most prevalent cause of cancer-related deaths worldwide [1, 2]. In Germany, the overall age-adjusted 5-year relative survival is $16.9 \%$, with considerable differences between men (15.5\%) and women (20.3\%) [3]. Cancer stage at the time of diagnosis is the most important predictor for treatment options, response to treatment and survival. Only 14\% of patients are diagnosed in stage I, since lung cancer in early stages is often asymptomatic [4]. The majority of lung tumours is diagnosed at the advanced stage IV (men: 72.4\%, women: $69.1 \%$ ) with a poor prognosis compared to other cancer diseases [3], which is often associated with high psychological, physical and social stress for patients and family caregivers [5]. Due to the poor prognosis, patients need to be aware of the limited life span to effectively engage in end-of-life decision making on advance care planning (ACP) under consideration of patient preferences [6].

To meet the challenge of coping with the complex needs of patients [7], an effective communication from the time of diagnosis between all involved persons (patients, family caregivers and healthcare providers) is essential [8]. The opportunity to communicate about the situation positively affects patients' quality of life [9]. Furthermore, communication is key to gathering important information, assisting patients in decision making and integrating palliative care early in the course of disease [10].

In spite of knowing about the positive effects of communication, patients, caregivers and healthcare providers often perceive communication skills of healthcare staff to be suboptimal and fragmented. Therefore, training of communication skills is recommended $[7,11]$ as well as a team approach to communication with patients, involving physicians and nurses. However, the actual application of communication skills in a team approach in routine practice remains difficult. It is therefore important to measure and improve its fidelity. The fidelity of any intervention is defined as the degree to which it is implemented as it was intended [12]. It reflects the adherence to content, frequency, duration and coverage of the planned approach [13]. Specific factors which are associated with intervention fidelity can be identified, such as aspects of environmental context, resources or the professional role understanding of healthcare professionals involved [14]. Insight into intervention fidelity and associated factors allows to tailor implementation strategies to the identified performance gaps and underlying causes [15].

For patients newly diagnosed with lung cancer stage IV, a structured longitudinal communication concept was developed and implemented to proactively address their complex care needs: The Heidelberg Milestones
Communication Approach (MCA) [7]. MCA aims at providing coherent care that integrates palliative care early and across the disease trajectory [7]. The concept includes four types of so-called milestone conversations (MCs) which involve patients, caregivers and an interprofessional tandem of physician and nurse navigator. MCs are scheduled at specific pivotal times with a thematic focus in the disease trajectory: Disclosure of diagnosis and prognosis (MC 1), Treatment with response or stability of disease (MC 2), Disease progression with reassessment of options and change or stop of diseasemodifying interventions (MC 3), and Transition to best supportive care and end-of-life consensus (MC 4). In follow-up sessions about a week after each $\mathrm{MC}$, the nurse calls patients to sustain communication [7]. Content and implementation of the MCA are described in detail in the manual (available (in German) upon request from the authors). The implementation of a new communication approach in routine care faces the challenges of behavioral changes in health professionals, adaptation of procedures and processes both within an organization and outside/between organizations [16]. For implementing the MCA, behavioral changes in health professionals are addressed in a compulsory training on interprofessional communication tailored to the specific conditions of the health care team in training. The training takes place once a month over a period of four months and includes an introduction of the concept and an on-thejob video-feedback. A shared routine documentation shall improve communication within the team [7]. Furthermore, necessary organizational changes (e.g. time slots and rooms) are addressed in the institution to facilitate team-based communication with patients.

Studies have shown that newly introduced concepts are adapted to routine care after implementation, thereby (at least partly) not adhering to the concept aim. Aspects perceived as helpful in routine care are consolidated, others abandoned. An assessment of key aspects after a consolidation period may elucidate approaches for further improvement of a concept [17].

The aim of this study is to assess the fidelity of the MCA approach after these implementation strategies, a process evaluation was conducted. The process evaluation focuses on contents (documented topics) in the MCs according to the manual both at the beginning of the implementation and after a consolidation period. Furthermore, we documented the frequency of topics addressed in the conversations.

\section{Methods}

The process evaluation was a prospective observational study with two assessment periods. Content analysis of patient files was used to evaluate implementation fidelity of the communication approach. All written records 
which are part of the routine documentation during MCs and follow-up calls were used for qualitative and quantitative analysis. Adherence to the manual was evaluated during the training period (t1: January to May 2018) and after six months (t2: September and October 2018). The first phase included five months, since there are fewer MCs conducted in the beginning that need to be integrated into clinical routine; the second phase included two months, when after implementation and routine use more MCs could be expected. Analysis focused on prognostic awareness, ACP and patient preferences.

\section{Setting}

The study took place in 2017 and 2018 on the chemotherapy outpatient department at the Thoracic Clinic, University Hospital Heidelberg, Germany. This hospital is a comprehensive cancer center and focuses on thoracic diseases, including lung cancer.

\section{Data collection}

All routine records collected by nurses in the observed periods were included in the data analysis. The nurses as part of the interprofessional tandem are responsible for the documentation of the routine records during MCs and follow-up calls. Five nurse navigators for oncology and palliative care and five physicians have completed the compulsory tailored training for interprofessional communication based on the MCA manual between December 2017 and March 2018. The training included the topics: communication theories, breaking bad news, physicianpatient communication, interprofessional communication, prognostic awareness, hope, SPIKES [18], NURSE [19] and shared decision making. The theoretical input was trained with simulated patients and as training on the job (observation and video feed-back of conversations with real patients). Additionally, the nurse navigators had a seven-day theoretical and practical briefing in October 2017, in which relevant aspects for nursing within the MCA were discussed. After the training, three nurse navigators conducted and documented the MCs together with physicians and the follow-up calls.

\section{Routine MC and follow-up call records}

Routine records are used for MCs and follow-up calls. They are partly standardized thus allowing the nurse navigators to document individually in free text. The standardised parts include information on date and duration of $\mathrm{MC}$ or follow-up call. The routine record for MCs also includes information about participants (physicians, nurse navigators, and family caregivers), number of MC (1-4 or other conversations) and current disease status (stable, unstable, debasing, and dying). The records for follow-up calls additionally include information on the use of the Integrated Palliative Care Outcome
Scale (IPOS) [20]. All aspects addressed by patients, physicians and nurses were documented in free text in the routine records. For analysis, contents of routine records were summarized on checklists for MCs (Additional file 1) and follow-up calls (Additional file 2). Checklists were qualitatively developed based on the aspects addressed in the manual of the MCA (Table 1).

\section{Checklist for MCs}

Data for analysis were extracted from the records using self-developed checklists. An aspect addressed in the free text part of the MC records was documented on the checklist as one of six general or of three MC-specific topics (Additional file 1).

"General topics of the MCA" include six aspects that are not linked to a particular MC, but are mentioned as either generally important (therapy, patient preferences, physical condition, psychological condition) or are not specifically described in the manual of the MCA (organisation, complementary medicine) (Additional file 1), but emerged from the $\mathrm{MC}$ records at the beginning of $\mathrm{MCA}$ implementation as frequently discussed. Additional file 3 (supplementary material) includes a detailed description of aspects and examples.

The MC-specific topics classify the process within the trajectory of a metastatic disease with a limited prognosis (Additional file 1). MC 1 focuses on patients' understanding of the disease, thus, diagnosis, further diagnostics procedures and prognosis should be addressed. At MC 2, in the stable situation of the disease, prognostic awareness and ACP should be addressed to prepare for the case of progression. For MC 3, tumour progression and the facilitation of prognostic awareness are paramount. MC 4 focuses on the transition to best supportive care as all possible disease-modifying interventions are exhausted including patients preferences [11]. Additional file 3 (supplementary material) includes a detailed description of aspects and examples.

\section{Checklist for follow-up calls}

For the checklist of follow-up calls no specific aspects are given in the manual, but the use of the Integrated Palliative Care Outcome Scale (IPOS) is recommended, which measures palliative care needs for patients and their families [20]. The checklist for follow-up calls initially consisted of the following standardised information: use of the Integrated Palliative Outcome Scale (IPOS), duration of the follow-up call, participating person, and date. The checklist was further developed based on frequently addressed aspects in the follow-up calls documented in the records at the beginning of MCA implementation. These aspects could be subsumed under six topics (Additional file 2) "Physical condition" and "Psychological condition" (both based on the IPOS), 
Table 1 Specific topics of the MCA manual

\begin{tabular}{|c|c|c|}
\hline MCs & Period in disease trajectory & Specific topics of the manual \\
\hline I & Diagnosis & $\begin{array}{l}\text { - Warn patient that there is bad news (SPIKES) } \\
\text { - Open and honest statement of diagnosis and prognosis } \\
\text { - Discuss further procedure (diagnostic, therapy) } \\
\text { - Show limitations of therapy options }\end{array}$ \\
\hline$\|$ & Stable disease & $\begin{array}{l}\text { - Summarize last conversation } \\
\text { - Clarify how patients think about the disease (Prognostic awareness) } \\
\text { - Use stable disease to talk about advance care planning regarding end-of-life care. } \\
\text { - Define precautionary authorized representative } \\
\text { - Advance care planning can also be discussed later (depends of patient's psychological condition) }\end{array}$ \\
\hline III & Tumour progression & $\begin{array}{l}\text { - Communicate tumour progression } \\
\text { - Check patient preferences } \\
\text { - Define new therapy goals regarding quality of life } \\
\text { - Bring in palliative care offers (IPOS) } \\
\text { - Question Prompt List }\end{array}$ \\
\hline IV & Best supportive care & $\begin{array}{l}\text { - Communicate the end of tumour centred treatment options } \\
\text { - Transition to best supportive care } \\
\text { - Include always patient preferences }\end{array}$ \\
\hline General topics & In every conversation & $\begin{array}{l}\text { - Organisation } \\
\text { - Physical condition } \\
\text { - Psychological condition } \\
\text { - Therapy } \\
\text { - Complementary medicine } \\
\text { - Patient preferences }\end{array}$ \\
\hline
\end{tabular}

"Medical care", "Nursing care", "Outpatient providers", and "Wishes and hopes" (emerged from records). Additional file 4 (supplementary material) includes a detailed description of aspects and examples.

\section{Data analysis}

All routine MC and follow-up call records were anonymized prior to analysis. Content of records was analysed quantitatively: For each record, incidences of aspects on the respective checklist were entered into a data matrix and further analysed using IBM SPSS version 24. Aspects were summarized to respective topics. A topic was considered as "addressed" if at least one aspect of the topic was documented. Absolute and relative frequencies of topics are reported for both assessment periods. For consolidation, frequencies of the main topics ACP, prognostic awareness and patient preferences in the two assessment periods were compared using Chi square tests.

\section{Results}

\section{MCs}

In the first assessment period (t1), $133 \mathrm{MCs}$ were carried out: $27 \mathrm{MC} 1$ for disclosure of diagnosis and prognosis, 51 MCs 2 for the treatment with response or stability of disease, $30 \mathrm{MC} 3$ discussing disease progression with reassessment of options and change or stop of disease-modifying interventions, two $\mathrm{MC} 4$ for the transition to best supportive care and end-of-life consensus, 14 other conversations without a special thematic focus and 9 conversations without a documentation of the MC (Table 2). The mean average duration of the $\mathrm{MC}$ was $30 \mathrm{~min}(\mathrm{SD}=10.7$; range: 20-60 min). Each nurse navigator documented an approximately equal share of the routine records.

In the second assessment period (t2), $172 \mathrm{MCs}$ were documented: $18 \mathrm{MC} \mathrm{1,} 47 \mathrm{MC} \mathrm{2,} 9 \mathrm{MC} \mathrm{3}$, and $1 \mathrm{MC} 4$. $42.3 \%$ of conversations (compared to $11 \%$ during $\mathrm{t} 1$ ) were not specified with an MC number: 26 other conversations without a special thematic focus and 71 conversations without documentation of the $\mathrm{MC}$ number (Table 2). There was no MC with a patient who was documented to be close to death. The mean duration of the $\mathrm{MCs}$ was $28 \mathrm{~min}$ ( $\mathrm{SD}=5.1$; range: 5-50 $\mathrm{min}$ ). The documentation of the routine records was not distributed equally among the nurse navigators. One of the nurse navigators documented a larger share than the two other nurse navigators.

\section{General topics}

General topics were analysed for all types of MCs. Patients' preferences were documented in all conversations up to a maximum of $27 \%(n=8)(\mathrm{AP} 1)$ and $23 \%(n=11)$ (AP2). The aspect "Organisation" was documented in 55$82 \%$ of the MCs during $\mathrm{t} 1$ and in $64-94 \%$ of the MCs during $\mathrm{t} 2$. Therapies were also written down in a majority of the MCs in both assessment periods ( $\mathrm{t} 1:$ 79-98\%, $\mathrm{t} 2$ : 67$89 \%)$. Psychological conditions were less often attended to during t2 (39-56\%, t1: 64-77\%). (Table 2).

\section{Milestone-specific topics}

In $\mathrm{MC} 1$, communication about diagnoses more often occurred during t2 $(50 \%(n=9)$, t1: $21 \%(n=6))$. 
Table 2 Thematic focuses of ME - period comparison

\begin{tabular}{|c|c|c|c|}
\hline & $\mathrm{t} 1(n=133)$ & t2 $(n=172)$ & $\begin{array}{l}\boldsymbol{p} \text {-value/ direction change } \\
\text { (increase/decrease) }\end{array}$ \\
\hline Milestone conversation (MC 1): & $\begin{array}{l}\text { N (\%) } \\
27(100)\end{array}$ & $\begin{array}{l}N(\%) \\
18(100)\end{array}$ & \\
\hline Diagnosis & $6(21)$ & $9(50)$ & $.04 \uparrow$ \\
\hline Further diagnostics & $12(43)$ & $8(44)$ & .92 \\
\hline Prognosis & $5(18)$ & $3(17)$ & .92 \\
\hline Organisation & $23(82)$ & $17(94)$ & $.23 \uparrow$ \\
\hline Physical condition & $17(61)$ & $13(72)$ & $.42 \uparrow$ \\
\hline Psychological condition & $18(64)$ & $7(39)$ & $.09 \downarrow$ \\
\hline Therapy & $22(79)$ & $16(89)$ & $.37 \uparrow$ \\
\hline Complementary medicine & $1(4)$ & Undocumented & $<.01$ \\
\hline Patient preferences & $4(14)$ & Undocumented & .09 \\
\hline Milestone conversation (MC 2): & $\begin{array}{l}N(\%) \\
51(100)\end{array}$ & $\begin{array}{l}N(\%) \\
47(100)\end{array}$ & \\
\hline Prognostic Awareness & $16(31)$ & $22(47)$ & $.12 \uparrow$ \\
\hline Advance Care Planning & $13(26)$ & $6(13)$ & $.11 \downarrow$ \\
\hline Organisation & $28(55)$ & $30(64)$ & $.37 \uparrow$ \\
\hline Physical condition & $28(55)$ & $39(83)$ & $<.01 \uparrow$ \\
\hline Psychological condition & $35(69)$ & $24(51)$ & $.08 \downarrow$ \\
\hline Therapy & $50(98)$ & $36(77)$ & $<.01 \downarrow$ \\
\hline Complementary medicine & $4(8 \%)$ & $3(6)$ & .78 \\
\hline Patient preferences & $7(14)$ & $11(23)$ & $.22 \uparrow$ \\
\hline Milestone conversation (MC 3): & $\begin{array}{l}N(\%) \\
30(100)\end{array}$ & $\begin{array}{l}N(\%) \\
9(100)\end{array}$ & \\
\hline Prognostic Awareness & $16(53)$ & $5(56)$ & .91 \\
\hline Advance Care Planning & $9(30)$ & $2(22)$ & .65 \\
\hline Tumour progression & $21(70)$ & $7(78)$ & .65 \\
\hline Organisation & $17(57)$ & $7(78)$ & $.25 \uparrow$ \\
\hline Physical condition & $20(67)$ & $7(78)$ & $.53 \uparrow$ \\
\hline Psychological condition & $23(77)$ & $5(56)$ & $.22 \downarrow$ \\
\hline Therapy & $29(97)$ & $6(67)$ & $<.01 \downarrow$ \\
\hline Complementary medicine & $1(3)$ & Undocumented & .58 \\
\hline Patient preferences & $8(27)$ & $1(11)$ & $.33 \downarrow$ \\
\hline Milestone conversation (ME 4): & $\begin{array}{l}N(\%) \\
2(100)\end{array}$ & $n=1$ & \\
\hline Best supportive care & $2(100)$ & Undocumented & \\
\hline Therapy (no further) & $1(50)$ & Undocumented & \\
\hline Other conversations & $\begin{array}{l}N(\%) \\
14(100)\end{array}$ & $\begin{array}{l}N(\%) \\
71(100)\end{array}$ & \\
\hline Prognostic Awareness & Undocumented & $17(23)$ & .05 \\
\hline Advance Care Planning & $3(21)$ & $24(32)$ & $.41 \uparrow$ \\
\hline Organisation & $12(86)$ & $51(69)$ & $.20 \downarrow$ \\
\hline Physical condition & $8(57)$ & $48(65)$ & .58 \\
\hline Psychological condition & $7(50)$ & $38(51)$ & .93 \\
\hline Therapy & $9(64)$ & $36(49)$ & $.28 \downarrow$ \\
\hline Complementary medicine & $2(14)$ & $4(5)$ & .23 \\
\hline
\end{tabular}


Table 2 Thematic focuses of ME - period comparison (Continued)

\begin{tabular}{llll}
\hline & $\mathrm{t} 1(n=133)$ & $\mathrm{t}$ ( $(n=172)$ & $\begin{array}{c}\boldsymbol{p} \text {-value/ direction change } \\
\text { (increase/decrease) }\end{array}$ \\
\hline Patient preferences & Undocumented & $17(23)$ & .05 \\
Conversations without ME number & $\mathbf{N}(\%)$ & $\mathbf{N}(\%)$ & $\mathbf{2 6 ( 1 0 0 )}$ \\
Prognostic Awareness & $\mathbf{9 ( 1 0 0 )}$ & $5(19)$ & $.39 \downarrow$ \\
Advance Care Planning & $3(33)$ & $4(15)$ & .64 \\
Organisation & $2(22)$ & $15(58)$ & $.02 \downarrow$ \\
Physical condition & $9(100)$ & $18(69)$ & $.06 \downarrow$ \\
Psychological condition & $9(100)$ & $12(56)$ & $.93 \uparrow$ \\
Therapy & $4(44)$ & $15(58)$ & $.02 \downarrow$ \\
Complementary medicine & $9(100)$ & $3(12)$ & .92 \\
Patient preferences & $1(11)$ & $5(19)$ & .58 \\
\hline
\end{tabular}

Prognosis was recorded in less than $20 \%$ of the conversations (t1: $18 \%(n=5), \mathrm{t} 2: 17 \%(n=3))$. For MC 2 and MC 3, the discussion of prognostic awareness and advance care planning was paramount. Prognostic awareness was more often documented in MC 3 (t1: $53 \%(n=$ 16), $\mathrm{t} 2: 56 \%(\mathrm{n}=5))$ than in MC $2(\mathrm{t} 1: 31 \%(\mathrm{n}=16), \mathrm{t} 2$ : $47 \%(n=22))$. ACP was written down up to $30 \%(n=9)$ of the conversations during t1 (MC 3, MC 2: 26\% ( $n=$ $13)$ ), but less often during t2 (MC 2: 13\% $(n=6)$, MC 3: $22 \%(n=2))$. During t2, only one MC 4 was carried out. The documentation included no milestone-specific topics. (Table 2).

\section{Follow-up calls}

During t1, 54 follow-up calls were conducted, which had a range of duration from five to $55 \mathrm{~min}$. The average duration of follow-up calls was 19 min. During t2, 92 follow-up calls were documented, which had a range of duration from ten to $45 \mathrm{~min}$. The average duration was $17 \mathrm{~min}$.

In most follow-up calls, the physical condition of the patients was recorded (t1: $82 \%(n=44), \mathrm{t} 2: 83 \%(n=$ 76)). Patients also occasionally brought up the topics of prognostic awareness (t1: 39\% $(n=21), \mathrm{t} 2: 24 \%(n=22))$ and of advance care planning (t1:17\% $(n=9)$, t2: $9 \%$ $(n=8))$. (Table 3$)$.

\section{Discussion}

To assess to what extent the MCA is implemented in practice, a process evaluation was conducted. For an assessment of intervention fidelity, healthcare providers' adherence to the manual regarding content of milestone conversations and follow-up calls was mixed. ACP was documented more often at January to May, while addressing prognostic awareness and documenting patient preferences were consolidated. Although in about half of the conversations these topics were not documented, we consider the documented conversations on key features of MCA as a major step towards its implementation as the communication concept was previously non-existent in this setting.

These results can be discussed in relation to multiple definitions of fidelity in the literature [21]. It provides a framework for discussing key features, but it is not necessary that each feature is included in all conversations as these need to be tailored to individual patient needs. According to Rietjens et al. [22], prognostic awareness is a complex issue with no internationally standardised form of survey or documentation. Furthermore, ACP lacks an international consensus on its definition. Nevertheless, in the MCA manual, existing recommendations that ACP in the palliative setting should be carried out as a communication process on the basis of individual readiness were included [22].

In a comparable study, the effectiveness of a nurse-led primary palliative care intervention was evaluated using visit checklists and visit audio-recordings to monitor adherence to intervention. Therefore, the intervention was divided into several clear defined components. For all components the fidelty was over $80 \%$ [23]. The difference with our study may be caused by a different method for assessing intervention fidelity, by the focus on routine clinical practice, by differences in the situation at baseline, or by other differences. Comparison of the studies is difficult. Another study using a systematic assessment procedure to report on implementation fidelity showed that $70 \%$ of all strategies were implemented with integrity [24]. However, this study focussed on the implementation strategy and not the intervention targeted at patients. In the MCA project the plan docheck-act-cycle was included as a measure to monitor implementation [7]. It was decided to rely on the routine clinical documentation to reflect the real-world setting as close as possible. This could be an explanation why it 
Table 3 Most documented topics of the follow-up calls - period comparison

\begin{tabular}{|c|c|c|c|}
\hline Topics documented & t1 & t2 & $\boldsymbol{p}$-value \\
\hline Total of records & $\begin{array}{l}\text { N (\%) } \\
54(100)\end{array}$ & $\begin{array}{l}\text { N (\%) } \\
92(100)\end{array}$ & \\
\hline Physical condition & $44(82)$ & $76(83)$ & .86 \\
\hline - General condition & $\cdot 18(33)$ & $\cdot 33(36)$ & .76 \\
\hline - Respiration & $\cdot 18(33)$ & $\cdot 13(14)$ & $<.01$ \\
\hline - Mobility & $\cdot 18(33)$ & $\cdot 13(14)$ & $<.01$ \\
\hline - Pain & $\cdot 18(33)$ & $\cdot 22(24)$ & .22 \\
\hline - Food \& Beverarge & $\cdot 16(30)$ & $\cdot 24(26)$ & .64 \\
\hline - Skin \& Hair & $\cdot 14(26)$ & $\cdot 18(20)$ & .37 \\
\hline • Sleep & $\cdot 9(17)$ & $\cdot 8(9)$ & .15 \\
\hline - Weight & • $5(9)$ & $\cdot 1(1)$ & .02 \\
\hline - Excretion & $\cdot 4(7)$ & $\cdot 11(12)$ & .38 \\
\hline - Nausea & $\cdot 2(4)$ & $\cdot 5(5)$ & .64 \\
\hline - Burden of symptoms & - Undocumented & $\cdot 7(8)$ & .04 \\
\hline Psychological condition & $37(69)$ & $70(76)$ & .32 \\
\hline - Prognostic Awareness & $\cdot 21(39)$ & $\cdot 22(24)$ & .06 \\
\hline - Positiv feelings & $\cdot 18(33)$ & $\cdot 28(30)$ & .72 \\
\hline - Burden/excessive demand & $\cdot 8(15)$ & $\cdot 18(20)$ & .47 \\
\hline - Family/Friends & $\cdot 7(13)$ & $\cdot 11(12)$ & .86 \\
\hline - Sport as motivation & $\cdot 5(9)$ & $\cdot 11(12)$ & .92 \\
\hline - Relationship problems & $\cdot 5(9)$ & $\cdot 7(8)$ & .73 \\
\hline • Fears & $\cdot 3(6)$ & $\cdot 7(8)$ & .64 \\
\hline - Unrest & $\cdot 2(4)$ & $\cdot 1(1)$ & .28 \\
\hline - Listlessness & $\cdot 2(4)$ & $\cdot 3(3)$ & .89 \\
\hline Medical care & $39(72)$ & $60(65)$ & .38 \\
\hline - Oral medication & $\cdot 15(28)$ & • $30(33)$ & .54 \\
\hline • Chemotherapy & $\cdot 12(22)$ & $\cdot 21(23)$ & .93 \\
\hline - General therapy & $\cdot 8(15)$ & $\cdot 11(12)$ & .59 \\
\hline • Radiotherapy & $\cdot 6(11)$ & $\cdot 11(12)$ & .88 \\
\hline - Inpatient admission & $\cdot 6(11)$ & • $3(3)$ & .06 \\
\hline - Laboratory & • $5(9)$ & $\cdot 7(8)$ & .73 \\
\hline$\cdot C T$ & $\cdot 4(7)$ & $\cdot 6(7)$ & .84 \\
\hline - Rehabilitation & $\cdot 3(6)$ & $\cdot 2(2)$ & .28 \\
\hline$\cdot \mathrm{MRT}$ & $2(4)$ & $\cdot 4(4)$ & .85 \\
\hline - Medical operation & $1(2)$ & - Undocumented & .19 \\
\hline Nursing care & $13(24)$ & $16(17)$ & .33 \\
\hline - Home care/Social services & $\cdot 7(13)$ & $8(9)$ & .19 \\
\hline - Care by family members & $\cdot 6(11)$ & $8(9)$ & .63 \\
\hline • Self-supply & - Undocumented & $3(3)$ & .18 \\
\hline Advance Care Planning & $9(17)$ & $8(9)$ & .15 \\
\hline • SAPV & $\cdot 7(13)$ & $4(4)$ & .06 \\
\hline - Patient decree & $\cdot 2(4)$ & $4(4)$ & .85 \\
\hline - Precautionary power & $\cdot 1(2)$ & $1(1)$ & .70 \\
\hline Outpatient providers & $6(11)$ & $11(12)$ & .88 \\
\hline
\end{tabular}


Table 3 Most documented topics of the follow-up calls - period comparison (Continued)

\begin{tabular}{|c|c|c|c|}
\hline Topics documented & t1 & t2 & $\boldsymbol{p}$-value \\
\hline - General practitioner & $5(9)$ & $8(9)$ & .91 \\
\hline - Psychological service & $1(2)$ & $3(3)$ & .62 \\
\hline - Radiation & Undocumented & $1(1)$ & .44 \\
\hline Wishes/Hopes/Attitude to life & $9(17)$ & $14(15)$ & .82 \\
\hline - Vacation & $\cdot 5(9)$ & • $9(10)$ & .92 \\
\hline - Improvement of health & $\cdot 4(7)$ & $\cdot 4(4)$ & .43 \\
\hline - Family events & - Undocumented & $\cdot 2(2)$ & .28 \\
\hline Appointments & $30(56)$ & $52(56)$ & .91 \\
\hline IPOS used & $54(100)$ & $92(100)$ & \\
\hline - Yes & $22(41)$ & $42(46)$ & \\
\hline$\cdot$ No & $32(59)$ & $50(54)$ & \\
\hline
\end{tabular}

seems that fidelity remained on a lower level than in other studies as communication tends to be less well recorded than, for instance, test results and treatments. Future research of the concept may use a more rigorous monitoring of the intervention fidelity, such as direct observation or analysis of audiotaped conversations.

ACP and prognostic awareness were defined as specific topics of MC 2. The aspect of patient preferences was not tied to a particular MC. However, these key features are interdependent and important for every MC. Inaccurate prognostic awareness may hinder ACP and the consistency of care with patients' goals [25]. Especially patients with advanced cancer need sufficient information for making realistic health-care decisions at the end of life. Studies have found a significant relationship between accurate prognostic awareness and reduced psychological distress in patients with advanced cancer [26]. Therefore, prognostic awareness takes on a high significance in the context of early palliative care. It has already been shown that well-informed patients express the wish for an early transition to palliative care in order to avoid aggressive therapies at the end of life [27]. The presence of prognostic awareness in turn increases the willingness to accept aspects of ACP. Conversely, ACP is an important strategy to improve end-of-life communication between patients and healthcare providers. Other potential benefits are that ACP allows patients to maintain a sense of control and gives them the opportunity to incorporate personal preferences [28].

Despite the relatively successful start of the implementation of the MCA, it should be considered how its implementation can be enhanced. The high emotional treshold in professionals for addressing these topics is well known [29]. Therefore, oncological societies emphasize the need to strenghten communication skills [30]. A communication training as this may be the initiation of a process that seeks for a change in behaviour and attitude. It seems likely that a longer follow-up would show higher fidelity [31]. On the other hand, a proportion of the trained health care professionals has been replaced by others as a consequence of planned rotations and other factors. It is a challenge to adapt the concept to practice while maintaining the three key features to all MCs which are important to support stage IV lung cancer patients.

Additionally, adherence to the MCA concept may be strenghtened by using a more rigorous plan to monitor adherence to the intervention and by providing support with additional training sessions and coaching for effective documentation. After a four-months training period with two theoretical and two practical on-the-job training sessions, only up to $50 \%$ of the relevant aspects are documented in the actual patient conversations. Furthermore, various conversations were documented as "Milestone conversations" without defining the precise phase in the disease trajectory. There might be several reasons which have to be further explored to respond accordingly.

Firstly, the performed training is only one aspect of dealing with relevant barriers to change in routine care. Implementation of the MCA concept meets with logistic challenges in a busy clinic setting. For instance, the physician and the nurse need to be available simultaniously as well as a protected room for the conversation. The role of local clinical opinion leaders seems crucial to keep the topic high on the agenda. Since behavioural change needs time and continuous support, "booster training sessions" might help to consolidate behaviour and knowledge (including phase definitions [32]) according to the MCA and facilitate routines [8]. Secondly, as indicated above, it is possible that topics were covered but not documented. Documentation by nurse navigators in communication is not standardized, but actually put down in plain free text. Out of the routine records now analysed, a documentation sheet could be 
developed to facilitate documentation. This might also foster interprofessional team communication and enhance good quality of patient care.

Thirdly, health care professionals may perceive milestone conversations as protected time with their patients, in which they are allowed to extend the usual consultation time. This might be a reason for the high percentage of "other conversations" after implementation of the MCA. Additionally, Milestone conversations stretched over multiple sessions, thus allowing for relationship building especially at the beginning of patient contact. Nevertheless, that could have led to a spread of content over multiple sessions and a fragmented documentation. The high number of aspects of physical condition documented may also be a sign for the respective high burden of this patient group and the priorities set by the patients concerning their needs.

Follow-up calls are seen as a useful tool to strengthen the key features of the concept. This way of communication serves not only to strengthen the relationship between patient and health care professionals, but also to clarify patient preferences. The possibility of having contact with the treatment team outside of planned MCs helps the patient to clarify his or her own status with regard to relevant information for ACP and prognostic awareness. Within the follow-up calls, nurse navigators provide emotional support to individuals tailored to patients' unique needs. Furthermore, the follow-up calls offer the possibility to develop individual strategies that support the patient regarding prognostic awareness and ACP [33].

\section{Strenghts and limitations}

The study is embedded in routine clinical practice and has therefore a high transferability of findings, although only one hospital was involved. The limitations of routine clinical documentation has already been highlighted. On the basis of the available documentation, we cannot assess contextual determinants of intervention fidelity [34]. The implementation activities heavily focused on training of health care professionals and logistic problems were addressed only after the first experiences. Finally, the data do not allow the analysis of individual patients' pathways as the documented conversations could not be linked to individual patients. Patient relevant outcomes are evaluated within a current randomized controlled trial, though, and will be shortly available.

\section{Conclusion}

The MCA provides a communication concept that oncologists and nurses can use to strengthen lung cancer patients' attitudes towards prognostic awareness, ACP and patients preferences. The study shows that a major step towards its implementation in routine clinical practice was made, but also that room for further improvement exists. To support implementation of the concept, adjustments to the concept based on the process evaluation are needed. Further work should investigate factors that support and detract from intervention fidelity to improve it in the future.

\section{Supplementary information}

Supplementary information accompanies this paper at https://doi.org/10. 1186/s12904-020-0527-1.

Additional file 1. Checklist milestone conversations: quantitative developed checklist for the analysis of the MCs.

Additional file 2. Checklist for follow-up calls: quantitative developed checklist for the analysis of follow-up calls.

Additional file 3. Relevant term definitions MCs: Contains the definition of relevant terms from the checklist of MCs and explains them using a suitable example.

Additional file 4. Relevant term definitions Follow-up calls: Contains the definition of relevant terms from the checklist of Follow-up calls and explains them using a suitable example.

\section{Abbreviations}

ACP: Advance Care Planning; IPOS: Integraded Palliative Outcome Scale; MC: Milestone Conversation; MCA: Milestone Communication Approach

\section{Acknowledgements}

We would like to thank all the patients who participated. We also gratefully appreciate the participation of all physicians and nurse navigators in the study

\section{Authors' contributions}

JB, MW, MV and KK conceived and designed the study. JB developed the checklists, analysed the data and wrote a first draft of this paper. KK was responsible for the statistical support, critical intellectual advise regarding content aspects of the study and supported in finalizing this manuscript. J J and ND planned and carried out the communication training. MT, LH, AS and $\mathrm{CJ}$ contributed to the manuscript. All authors have given final approval of the final manuscript.

\section{Funding}

This research was funded by the Federal Ministry of Health, Germany (150454401), and the National Center for Tumor Diseases (NCT 3.0, G835), Heidelberg, Germany. The funder provided no role in the conception or design of this study, the acquisition, analysis, interpretation of data and drafting manuscript.

\section{Availability of data and materials}

The datasets used and analyzed during the current study are available from the corresponding author on reasonable request.

\section{Ethics approval and consent to participate}

This work is part of a larger study. The study protocol takes into account the evaluation of the routine records and follow-up records. Patients gave their written informed consent for participation and analysis of all collected data. The study was approved by the Ethics Committee of the University Hospital Heidelberg, Germany (protocol no. S-561/2017).

Consent for publication

Not applicable.

Competing interests

The authors declare that they have no competing interests. 


\section{Author details}

'Department of General Practice and Health Service Research, University Hospital Heidelberg, Im Neuenheimer Feld 130.3, 69120 Heidelberg, Germany. ${ }^{2}$ Department of Thoracic Oncology, Thoraxklinik Heidelberg, Heidelberg University Hospital, Röntgenstraße 1, D-69126 Heidelberg, Germany. ${ }^{3}$ Institute of Medical and Pharmaceutical Proficiency Assessment, Malakoff Passage, Rheinstraße 4, D-55116 Mainz, Germany.

Received: 5 June 2019 Accepted: 14 February 2020

Published online: 18 February 2020

\section{References}

1. Cheng T-YD, Cramb SM, Baade PD, Youlden DR, Nwogu C, Reid ME. The international epidemiology of lung cancer: latest trends, disparities, and tumor characteristics. J Thorac Oncol. 2016;11(10):1653-71.

2. Grønberg BH, Sundstrøm S, Kaasa S, Bremnes RM, Fløtten $\varnothing$, Amundsen T, Hjelde HH, von Plessen C, Jordhøy M. Influence of comorbidity on survival, toxicity and health-related quality of life in patients with advanced nonsmall-cell lung cancer receiving platinum-doublet chemotherapy. Eur J Cancer. 2010;46(12):2225-34.

3. Eberle A, Jansen L, Castro F, Krilaviciute A, Luttmann S, Emrich K, Holleczek B, Nennecke A, Katalinic A, Brenner H. Lung cancer survival in Germany: a population-based analysis of 132,612 lung cancer patients. Lung Cancer. 2015;90(3):528-33.

4. Grose D, Morrison DS, Devereux G, Jones R, Sharma D, Selby C, Docherty K, McIntosh D, Nicolson M, McMillan DC. The impact of comorbidity upon determinants of outcome in patients with lung cancer. Lung Cancer. 2015; 87(2):186-92.

5. Yanwei L, Dongying L, Zhuchen $Y$, Ling L, Yu Z, Zhanyu P. A double-edged sword: should stage IV non-small cell lung cancer patients be informed of their cancer diagnosis? Eur J Cancer Care. 2017;26(6):e12665.

6. Epstein AS, Prigerson HG, O'Reilly EM, Maciejewski PK. Discussions of life expectancy and changes in illness understanding in patients with advanced cancer. J Clin Oncol. 2016;34(20):2398.

7. Siegle A, Villalobos M, Bossert J, Krug K, Hagelskamp L, Krisam J, Handtke V, Deis $N$, Jünger J, Wensing $M$. The Heidelberg milestones communication approach (MCA) for patients with prognosis< 12 months: protocol for a mixed-methods study including a randomized controlled trial. Trials. 2018; 19(1):438.

8. Barth J, Lannen P. Efficacy of communication skills training courses in oncology: a systematic review and meta-analysis. Ann Oncol. 2010;22(5): 1030-40.

9. Moore PM, Rivera S, Bravo-Soto GA, Olivares C, Lawrie TA. Communication skills training for healthcare professionals working with people who have cancer. Cochrane Database Syst Rev. 2018;7.

10. Baile WF, Aaron J. Patient-physician communication in oncology: past, present, and future. Curr Opin Oncol. 2005;17(4):331-5.

11. Villalobos M, Coulibaly K, Krug K, Kamradt M, Wensing M, Siegle A, Kuon J, Eschbach C, Tessmer G, Winkler E. A longitudinal communication approach in advanced lung cancer: a qualitative study of patients', relatives' and staff's perspectives. Eur J Cancer Care. 2018;27(2):e12794.

12. Slaughter SE, Hill JN, Snelgrove-Clarke E. What is the extent and quality of documentation and reporting of fidelity to implementation strategies: a scoping review. Implement Sci. 2015;10(1):129.

13. Willeboordse F, Schellevis F, Meulendijk M, Hugtenburg J, Elders P. Implementation fidelity of a clinical medication review intervention: process evaluation. Int J Clin Pharm. 2018:40(3):550-65.

14. Atkins L, Francis J, Islam R, O'Connor D, Patey A, Ivers N, Foy R, Duncan EM, Colquhoun $\mathrm{H}$, Grimshaw JM. A guide to using the theoretical domains framework of behaviour change to investigate implementation problems. Implement Sci. 2017;12(1):77.

15. Richards DA. The complex interventions framework. In: Complex interventions in health. Routledge; 2015. p. 27-41.

16. Grol R, Grimshaw J. From best evidence to best practice: effective implementation of change in patients' care. Lancet. 2003;362(9391):1225-30.

17. Baumann A, Cabassa L, Wiltsey Stirman S. Adaptation in dissemination and implementation science. In: Dissemination and implementation research in health: translating science to practice, vol. 2; 2017. p. 286-300.

18. Baile WF, Buckman R, Lenzi R, Glober G, Beale EA, Kudelka AP. SPIKES—a sixstep protocol for delivering bad news: application to the patient with cancer. Oncologist. 2000;5(4):302-11.
19. Fischer GS, Tulsky JA, Arnold RM. Communicating a poor prognosis. Topics in palliative care. 2000;4:75-94.

20. Holland J, Watson M, Dunn J. The IPOS new international standard of quality Cancer care: integrating the psychosocial domain into routine care. Psychooncology. 2011;20(7):677-80.

21. Cutbush S, Gibbs D, Krieger K, Clinton-Sherrod M, Miller S. Implementers' perspectives on Fidelity of implementation. Health Promot Pract. 2017;18(2): 275-82.

22. Rietjens JA, Sudore RL, Connolly M, van Delden JJ, Drickamer MA, Droger M, van der Heide A, Heyland DK, Houttekier D, Janssen DJ. Definition and recommendations for advance care planning: an international consensus supported by the European Association for Palliative Care. Lancet Oncol. 2017;18(9):e543-51.

23. Robbins-Welty GA, Mueser L, Mitchell C, Pope N, Arnold R, Park S, White D, Smith KJ, Reynolds C, Rosenzweig M. Interventionist training and intervention fidelity monitoring and maintenance for CONNECT, a nurse-led primary palliative care in oncology trial. Contemp Clin Trials commun. 2018; 10:57-61.

24. Richards Z, Kostadinov I, Jones M, Richard L, Cargo M. Assessing implementation fidelity and adaptation in a community-based childhood obesity prevention intervention. Health Educ Res. 2014;29(6):918-32.

25. Tang ST, Liu T-W, Liu LN, Chiu C-F, Hsieh R-K, Tsai C-M. Physician-patient end-of-life care discussions: correlates and associations with end-of-life care preferences of cancer patients - a cross-sectional survey study. Palliat Med. 2014;28(10):1222-30

26. Applebaum AJ, Kolva EA, Kulikowski JR, Jacobs JD, DeRosa A, Lichtenthal WG, Olden ME, Rosenfeld B, Breitbart W. Conceptualizing prognostic awareness in advanced cancer: a systematic review. J Health Psychol. 2014; 19(9):1103-19.

27. Janssens A, Teugels L, Kohl S, Michielsen T, Van Meerbeeck J. Integrating early palliative care (EPC) in the management of lung cancer: the role of the thoracic oncologist. Lung Cancer. 2015;90(2):135-8.

28. Zwakman M, Jabbarian L, van Delden J, van der Heide A, Korfage I, Pollock K, Rietjens J, Seymour J, Kars M. Advance care planning: a systematic review about experiences of patients with a life-threatening or life-limiting illness. Palliat Med. 2018;32(8):1305-21.

29. Shanafelt T, Dyrbye L. Oncologist burnout: causes, consequences, and responses. J Clin Oncol. 2012;30(11):1235-41.

30. Peppercorn JM, Smith TJ, Helft PR, DeBono DJ, Berry SR, Wollins DS, Hayes DM, Von Roenn JH, Schnipper LE. American society of clinical oncology statement: toward individualized care for patients with advanced cancer. J Clin Oncol. 2011:29(6):755-60.

31. Rogers EM. The innovation-decision process. Diffusion of innovations, vol. 5: 2003. p. $168-218$

32. Santacroce SJ, Maccarelli LM, Grey M. Intervention fidelity. Nurs Res. 2004; 53(1):63-6.

33. Desrochers F, Donivan E, Mehta A, Laizner AM. A psychosocial oncology program: perceptions of the telephone-triage assessment. Support Care Cancer. 2016;24(7):2937-44.

34. Hasson $\mathrm{H}$. Systematic evaluation of implementation fidelity of complex interventions in health and social care. Implement Sci. 2010;5(1):67.

\section{Publisher's Note}

Springer Nature remains neutral with regard to jurisdictional claims in published maps and institutional affiliations.

Ready to submit your research? Choose BMC and benefit from:

- fast, convenient online submission

- thorough peer review by experienced researchers in your field

- rapid publication on acceptance

- support for research data, including large and complex data types

- gold Open Access which fosters wider collaboration and increased citations

- maximum visibility for your research: over $100 \mathrm{M}$ website views per year

At $\mathrm{BMC}$, research is always in progress.

Learn more biomedcentral.com/submissions 